\title{
COMPLEMENTARY GENES CONTROLLING RESPIRATION OF YEAST AND THEIR RELATION TO THE STABILITY OF THE CYTOPLASMIC FACTOR
}

\author{
NORIO GUNGE \\ Dai-Nippon Sugar Manufacturing Co., Ltd., Yokohama
}

Received September 29, 1965

The presence of both a normal respiratory gene and the cytoplasmic factor is necessary for the complete development of a cytochrome system in yeast (Ephrussi 1953). The cytoplasmic factor is known to mutate spontaneously due to failure of its own autonomous duplication, independent of nuclear state. Some connection, however, seems to exist between the cytoplasmic factor and the nucleus, because the character of "unstable" strains producing respiration-deficient (RD) cells in high frequencies during vegetative growth segregates regularly as if controlled by a gene (Ephrussi and Hottinguer 1951).

This report will describe complementary genes controlling the respiratory competence of bakers' yeast, and will present data suggesting the loss or instability of the cytoplasmic factor associated with the mutation in these respiratory genes. Similar observations were recently reported by Sherman (1963), however, the present study has been accomplished independently.

\section{MATERIALS AND METHODS}

Sufficiency and deficiency of respiration in yeast : Sufficiency/deficiency of respiration in yeast was estimated by the positive/negative reaction in an acetate utilizationindicator color change test (Ogur 1954), the presence/absence of growth on the nonfermentable carbon sources, glycerol and lactate (Nagai and Nagai 1958, Ogur and St. John 1956) and the color differentiation with the TTC overlay technique (Nagai 1959). When necessary, the ability/inability of aerobically grown cells to absorb oxygen was measured by a Warburg's apparatus. Generally, inconsistency did not occur among these estimations, except that several RD strains produced pink color within $3 \mathrm{hr}$ on the plate overlaid with TTC agar.

Complementary restoration of respiration in crosses between $R D$ yeasts : $R D$ strains of opposite mating types were mated in a nutrient broth (glucose, peptone and yeast extract) according to Lindegren's mass mating technique. The mating mixture (48hr culture) containing numerous zygotes or cells from hybrid which was obtained by isolation of zygotes were subjected to the above estimations of respiration-sufficiency/ deficiency. 
The production of standard vegetative mutants : Standard vegetative RD mutants of mating types $a$ and $\alpha$ were obtained by acriflavine treatment of respiring haploids from the Carbondale breeding stock. They proved to have the normal genic constitution, for the crosses between each of them and a normal haploid gave only asci which produced four respiring progenies.

The presence or absence of the cytoplasmic factor $\left(\rho^{+}\right.$or $\left.\rho^{-}\right): \rho^{+} / \rho^{-}$in RD yeasts was judged from the presence/absence of complementary restoration of respiration in crosses of $\mathrm{RD}$ yeasts with the standard vegetative mutants.

The absorption spectrum : Cytochromes were detected for cells aerobically grown by reciprocal shaking in a Sakaguchi's bottle using a Cary recording spectrophotometer Model IS, with the aid of opalecent glass plate.

\section{RESULTS AND DISCUSSIONS}

1. Analysis of respiratory genes

It was shown in the previous paper (Gunge 1966) that diploid RD cells were produced in unusually high frequencies of about 10 30\% from tetraploid respiring bakers' yeasts (strain $\mathrm{H}$ and some crosses between respiring diploid offsprings from it). Later, it was further found that pairwise crossing of such RD segregants often resulted in the production of respiring tetraploids which, on meiosis, segregated $\mathrm{RD}$ yeasts again in frequencies of about $70 \%$. Judging from the fact that vegetative cultures of these respiring tetraploid yeasts contain only a few $\mathrm{RD}$ cells (about $1 \%$ or less), it is inferred that the frequent segregation of $\mathrm{RD}$ yeasts may result from a peculiar state of the nucleus. One plausibility is that complementary genes control the respiration of tetraploid yeasts under heterozygous constitution containing many recessive alleles. When meiosis occurs in such yeasts, one can expect that considerable numbers of diploid segregants become either homozygous or heterozygous for one or more recessive alleles of the complementary genes. In such homozygotes, there is a fair chance that segregants have non-allelic defects capable of producing respirationally competent hybrids by crossing. Respiring yeasts may be produced in asci which have resulted from the crosses between such RD segregants as the result of combination of non-allelic dominant genes separately involved in each of component RD maters. In the heterozygotes, segregants become respiring yeasts and $\mathrm{RD}$ segregants can be expected to appear again by combination of allelic recessive genes commonly involved in each of component respiring maters.

If the above assumption is correct, non-allelic RD yeasts must be frequently found among haploid progenies from the tetraploid yeasts producing RD segregants in unusually high frequencies. To substantiate this, the tests were made with five out of six surviving haploids from independent four-spored asci of diploid yeasts, 133b, 133c and 133d, which were three of four non-mater sister segregants in ascus No. 133 of 
tetraploid parent strain H (cf. Table 1 in Gunge 1966).

Table 1. Diagnosis of respiration-deficiency in haploids isolated from tetraploid strain of bakers' yeast

\begin{tabular}{|c|c|c|c|c|c|c|c|}
\hline Strain & Parent & $\begin{array}{l}\text { Mating } \\
\text { type }\end{array}$ & $\begin{array}{l}\text { Acetate } \\
\text { utili- } \\
\text { zation } \\
\text { test }\end{array}$ & $\begin{array}{l}\text { Growth } \\
\text { on non- } \\
\text { fermen- } \\
\text { table } \\
\text { carbons }\end{array}$ & $\begin{array}{c}\text { Color by } \\
\text { TTC, } \\
\text { overlaying }\end{array}$ & $\begin{array}{c}\mathrm{Qo}_{2} \\
(\mu \mathrm{l} / \mathrm{mg} \\
\text { dry } \\
\text { cell } / \mathrm{hr})\end{array}$ & $\rho$ \\
\hline$b-1$ & $133 \mathrm{~b}$ & $\alpha$ & - & - & white to faintly pink & 0 & - \\
\hline$c-1$ & $133 \mathrm{c}$ & $\alpha$ & - & - & white to faintly pink & 0 & - \\
\hline$d-1$ & $133 d$ & $a$ & - & - & white & 0 & + \\
\hline$d-2$ & $133 \mathrm{~d}$ & $a$ & - & - & white to faintly pink & 0 & + \\
\hline$d-3$ & $133 \mathrm{~d}$ & $\alpha$ & - & - & white to faintly pink & 0 & - \\
\hline \multicolumn{2}{|c|}{ Control (respiring) } & - & + & + & red (approx. 1\% white) & 90 & + \\
\hline
\end{tabular}

$+/-$ indicate the positive/negative reaction of acetate utilization test, the presence/absence of growth on glycerol and lactate media, and the presence/absence of the cytoplasmic factor.

Table 1 shows that all of these five haploids, (b-1), (c-1), (d-1), (d-2) and (d-3) are RD mutants. The absorption spectrum revealed that all of them contained cytochrome c, but lacked cytochrome a. Cytochrome b was absent in mutants (d-1), (d-2) and (d-3), but was faintly detectable in mutants $(b-1)$ and $(c-1)$. Since $\rho^{-}$RD mutant is generally accepted as deficient in both cytochromes $a$ and $b$, the results obtained with mutants (b-1) and (c-1) are of great interest, though more intensive and genetic examination remains for a future study. Among them, at least the two strains, (d-1) and (d-2), could be concluded to have a mutant state in nucleus, because they could not respire in despite of $\rho^{+}$. Each of the five mutants was crossed to a respiring standard haploid and the four-spored asci were analyzed. Segregation for respiration (sufficiency: deficiency) was normal (2:2) with the three mutants, (d-1), (d-2) and (d-3); and irregular $(2: 2,1: 3,0: 4)$ with the two, (b-1) and (c-1), the asci frequencies being 2,2 , and 8 in (b-1) and 1, 2, and 2 in (c-1). Diploid meiosis of the analyzed tetrads was confirmed by $2: 2$ segregation of the mating type and by the haploid size of segregant cells. Therefore, mutants $(\mathrm{d}-1),(\mathrm{d}-2)$ and $(\mathrm{d}-3)$ may be considered to be single gene mutants.

Irregular segregation in the cross, $(\mathrm{c}-1) \times$ respiring haploid, was analyzed as follows. Table 2 shows the result of tetrad analysis. Judging from the presence or absence of respiration before and after pairwise crossing between each other, segregants were found to be divided into four groups, $A, B, C$ and $D . \quad A$ includes respiring segregants; $B, \rho^{+}$ RD mutants; $C$ and $D, \rho^{-} \mathrm{RD}$ mutants characterized by the presence and absence, respectively, of respiration when crossed to $B$ ( $\rho^{+} \mathrm{RD}$ mutants). Complementary restoration of respiration between $\mathrm{RD}$ mutants was observed only in a part of zygotes between $B$ and $C$. As a rule, segregation for respiration (sufficiency: deficiency) in tetrads was $4: 0$ in crosses of $A \times A, 2: 2$ in crosses of $A \times B$ and $A \times C$, and irregular $(2: 2,1: 3,0: 4)$ in crosses of $B \times C$ and $A \times D$. Here again, indication of diploid meiosis, 
i. e. 2:2 segregation for the mating type and the haploid size of segregant cells, were observed in all analyzed tetrads. The possible explanation for the above results is that (1) at least two complementary genes (tentatively denoted as $R_{1} / r_{1}$ and $R_{2} / r_{2}$ ) control the respiration in the yeasts concerned, (2) genotypes of $A, B, C$ and $D$ are $R_{1} R_{2}, r_{1} R_{2}$, $R_{1} r_{2}$ and $r_{1} r_{2}$, respectively, and (3) thus the cross, (c-1) $\times$ respiring haploid is to be

Table 2. Tetrad analysis of cross, (c-1) $\times$ respiring haploid

\begin{tabular}{|c|c|c|c|c|c|}
\hline $\begin{array}{l}\text { Ascus } \\
\text { No. }\end{array}$ & Spore & $\begin{array}{l}\text { Mating } \\
\text { type }\end{array}$ & $\begin{array}{l}\text { Respi- } \\
\text { ration }\end{array}$ & $\rho$ & $\begin{array}{l}\text { Group- } \\
\text { ing }\end{array}$ \\
\hline \multirow{4}{*}{1} & $\mathrm{a}$ & $a$ & + & + & $A$ \\
\hline & $\mathrm{b}$ & $\alpha$ & - & - & $C$ \\
\hline & $\mathrm{c}$ & $a$ & - & - & $D$ \\
\hline & $\mathrm{d}$ & $\alpha$ & - & + & $B$ \\
\hline \multirow{4}{*}{2} & $\mathrm{a}$ & $a$ & - & - & $D$ \\
\hline & $b$ & $\alpha$ & + & + & $A$ \\
\hline & $\mathrm{c}$ & $a$ & + & + & $A$ \\
\hline & $\mathrm{d}$ & $\alpha$ & - & - & $D$ \\
\hline \multirow{4}{*}{3} & $\mathrm{a}$ & $a$ & - & + & $B$ \\
\hline & $\mathrm{b}$ & $a$ & - & - & $C$ \\
\hline & $c$ & $\alpha$ & - & - & $C$ \\
\hline & $\mathrm{d}$ & $\alpha$ & - & + & $B$ \\
\hline \multirow{4}{*}{4} & $\mathrm{a}$ & $a$ & - & - & $D$ \\
\hline & $\mathrm{b}$ & $\alpha$ & - & - & $C$ \\
\hline & $\mathrm{c}$ & $\alpha$ & + & + & $A$ \\
\hline & $d$ & $a$ & - & + & $B$ \\
\hline \multirow{4}{*}{5} & $\mathrm{a}$ & $\alpha$ & - & - & $C$ \\
\hline & $\mathrm{b}$ & $a$ & - & + & $B$ \\
\hline & $\mathrm{c}$ & $a$ & - & + & $B$ \\
\hline & $\mathrm{d}$ & $\alpha$ & - & - & $C$ \\
\hline
\end{tabular}

$+/-$ indicate the sufficiency/deficiency of respiration and the presence/absence of the cytoplasmic factor expressed as $r_{1} r_{2} \times R_{1} R_{2}$, giving irregular segregation.

Phenotypic deviations from this explanation are as follows: 3:1 was found in one out of 20 asci from $A \times$ $A, 1: 3$ in three out of 57 asci from $A$ $\times B, 3: 1$ in two and $1: 3$ in one out of 51 asci from $A \times C . \quad 3: 1$ asci in $A \times$ $C$ may arise from gene conversion (Lindegren 1955, Lindegren et al. 1956), or from super-numernary mitosis (Winge et al. 1950, 1954a 1954b, Winge 1957), judging from the fact that six or more spored asci were found, though not frequently. Other aberrant asci are accounted for by the presence of $\rho^{-} R_{1} R_{2}$ segregants. Respiration-deficiency in most zygotes of $B \times C$ is ascribable to the fact to be stated later that $\left(\rho^{+} \quad r_{1} R_{2}\right.$ mutants easily produced during vegetative growth many $\rho^{-}$cells which produced $\rho^{-}$ zygotes when crossed to group $C$ ( $\rho^{-}$RD mutants).

The suppressiveness (Eph-russi et al. 1955) due to $\rho^{-}$in group $C$ does not seem to work, for frequent production

of $\mathrm{RD}$ zygotes have not been observed in crosses of $C$ and $A$.

Irregular segregations in the cross, $(b-1) \times$ respiring haploid, may be explained as multiple genes segregation, just as in the result obtained with the cross, $(c-1) \times$ respiring haploid, although detailed analysis remains for a future study.

The genotypes of mutant (b-1) and of the segregational single gene mutants, (d-1), (d-2) and (d-3), were determined as follows. Supposing from the presence of respiration when crossed to mutants of genotype $r_{1} r_{2} \rho^{-}$, the $\rho^{+}$mutant (d-2) was concluded to owe its deficiency to mutation in a gene different from $R_{1}$ and $R_{2}$. Hence, gene $R_{3}$ controls 
the respiration together with $R_{1}$ and $R_{2}$ complementally. The genotype of (d-2) may be represented as $R_{1} R_{2} r_{3}$. The symbol $R_{3}$, therefore, should be added to each of the already analyzed genotypes, $R_{1} R_{2}, r_{1} R_{2} R_{1} r_{2}$ and $r_{1} r_{2}$. Mutant (d-1) was found to have genotype of $r_{1} R_{2} R_{3}$, because respiration was restored when crossed to either of the genotypes $R_{1} r_{2} R_{3}$ and $R_{1} R_{2} r_{3}$, but not when crossed to the genotype $r_{1} R_{2} R_{3}$.

$\rho^{-}$mutant (d-3) restored the respiration when crossed to either of $r_{1} R_{2} R_{3}$ and $R_{1} R_{2} r_{3}$, but not when crossed to $R_{1} r_{2} R_{3}$, which seems to be associated with $\rho^{-}$, as described later. Therefore, the genotype of (d-3) was expected to be $R_{1} r_{2} R_{3}$, or $R_{1} R_{2} R_{3} r_{4}$ on the assumption of further participation of complementary gene $R_{4}$ in the respiration. All of the 10 genic RD segregants from the cross of $(\mathrm{d}-3) \times$ respiring haploid were $\rho^{-}$. Judging from the nature which coincides with that of mutant (d-3), relating to the presence or absence of respiration after crossing with various mutants of known genotype, mutant (b-1) was concluded to have at least the two dominant genes $R_{1}$ and $R_{3}$. Accordingly, the possibility that multiple mutant genes may be involved in mutant (b-1) also suggests the participation of respiratory gene(s) besides $R_{1}, R_{2}$ and $R_{3}$ in the respiration.

It was thus established that many recessive alleles of respiratory genes are simultaneously contained in a given respiring strain of bakers' yeast. The reasons for the frequent production of RD segregants is supported by the converse result in another experiment which established that haploid progenies from a tetraploid yeast characterized by few production of $\mathrm{RD}$ segregants were mostly respiration-sufficient.

2. Instability of the cytoplasmic factor in genic mutants

It has been reported that $\rho^{+}$genic mutants sometimes revert to the wild type (Raut 1953, Yčas and Starr 1953). In the long course of experiments with the above-mentioned various mutants, it was found that the reversion of $\mathrm{RD}$ haploids to respiring ones (estimated by a mixture of red and white colonies on plates overlaid with TTC agar) occurred only in some strains of the genotypes $r_{1} R_{2} R_{3}$ and $R_{1} R_{2} r_{3}$, but never in strains of the genotype of $R_{1} r_{2} R_{3}$. Irreversible mutation of the latter strains was expected to be ascribable to the presence of recessive gene $r_{2}$ which appears to be associated with $\rho^{-}$, judging from the result of analysis of progenies from a cross, $(\mathrm{c}-1) \times$ respiring haploid.

To make clear the relation of $\rho^{+}$or $\rho^{-}$to each of the analyzed respiratory genes, examination was carried out for haploid segregants ( 2 dominants and 2 recessives) in the asci of the crosses, respiring haploid $\times$ several strains of $\mathrm{RD}$ yeasts which have genotypes $r_{1} R_{2} R_{3}, R_{1} r_{2} R_{3}$ and $R_{1} R_{2} r_{3}$. In such tetrads, $\rho^{-}$mutant strains among segregants of genotype $R_{1} R_{2} R_{3}$ were distinguished from genic mutants by the production of four respiring progenies in the asci obtained by crossing them with a respiring haploid. Results of the experiment were as follows. In dissection of 20 four-spored asci of 6 strains by $A \times C$, all of $40 \quad R_{1} r_{2} R_{3}$ segregants were associated with $\rho^{-}$, whereas $R_{1} R_{2} R_{3}$ segregants were produced in a ratio of $39 \rho^{+}: 1 \rho^{-}$. Dissecting 51 four-spored asci of 7 strains, produced by $A \times B$, gave $r_{1} R_{2} R_{3}$ and $R_{1} R_{2} R_{3}$ segregants, respectively, in ratios of $73 \rho^{+}: 29 \rho^{-}$and of $99 \rho^{+}: 3 \rho^{-}$, and 10 four-spored asci from $(\mathrm{d}-2) \times$ respiring haploid 
produced $R_{1} R_{2} r_{3}$ and $R_{1} R_{2} R_{3}$ segregants, respectively, in ratios of $17 \rho^{+}: 3 \rho^{-}$and of 19 $\rho^{+}: 1 \rho^{-}$. Only one $\rho^{-}$mutant strain was found among $80 R_{1} R_{2} R_{3}$ segregants from 20 four-spored asci of 3 strains produced between respiring haploids. Association of $r_{2}$ with $\rho^{-}$could be observed also in all of $r_{1} r_{2} R_{3}$ segregants from both crosses, $B \times C$ and $A \times D$, and therefore $r_{2} \rho^{-}$was concluded to be epistatic to $r_{1} \rho^{+}$.

It will be inferred from these that mutation of respiratory genes results in the loss or instability of the cytoplasmic factor. This view may be confirmed by the observation that the rate of production of $\rho^{-}$cells from $\rho^{+}$yeasts during vegetative growth was much higher in strains of genotypes $r_{1} R_{2} R_{3}$ and $R_{1} R_{2} r_{3}$ than in strains of genotype $R_{1} R_{2} R_{3}$, as shown in Table 3. In this table, the ratio of $\rho^{-} / \rho^{-}+\rho^{+}$in the cell population was determined by counting $\rho^{+}$and $\rho^{-}$colonies formed on plates in $40 \mathrm{hr}$ at $30^{\circ} \mathrm{C}$ following inoculation of cells from slant cultures. $\rho^{+}$and $\rho^{-}$were identified in $R_{1} R_{2} R_{3}$ strains by the TTC overlay technique, and in genic RD mutants by crossing sampled cells from each colony to the standard vegetative mutants. The frequent production of cytoplasmic $\mathrm{RD}$ mutants during vegetative growth was first observed in unstable respiring yeast (Ephrussi and Hottinguer 1951). It seems, however, that the character of unstable respiring yeast contributes little to the above results, because the instability of the

Table 3. Stability and instability of the cytoplasmic factor of vegetatively growing cells

\begin{tabular}{|c|c|c|c|c|}
\hline \multirow[t]{2}{*}{ Genotype } & \multirow[t]{2}{*}{ Strain } & \multicolumn{2}{|c|}{$\begin{array}{c}\text { Number of } \\
\text { colonies }\end{array}$} & \multirow{2}{*}{$\begin{array}{c}\text { Percent } \\
\text { of } \\
\rho^{-} \text {colonies }\end{array}$} \\
\hline & & & $\rho^{-}$ & \\
\hline \multirow[t]{6}{*}{$r_{1} R_{2} R_{3}$} & $1 \mathrm{~d}$ & 1 & 45 & 98 \\
\hline & $1 \mathrm{~d}^{*}$ & 0 & 48 & 100 \\
\hline & $3 a$ & 15 & 17 & 53 \\
\hline & $3 d$ & 2 & 22 & 92 \\
\hline & $4 \mathrm{~d}$ & 35 & 14 & 29 \\
\hline & $5 \mathrm{~b}$ & 32 & 22 & 41 \\
\hline \multirow[t]{6}{*}{$R_{1} R_{2} r_{3}$} & $d-2$ & 4 & 91 & 96 \\
\hline & $\mathrm{d}-2^{*}$ & 6 & 42 & 88 \\
\hline & $2 \mathrm{~F}-1 \mathrm{~d}^{* *}$ & 16 & 54 & 77 \\
\hline & $2 \mathrm{~F}-7 \mathrm{~d}^{* *}$ & 2 & 46 & 96 \\
\hline & $2 \mathrm{~F}-8 \mathrm{c} * *$ & 0 & 48 & 100 \\
\hline & $2 \mathrm{~F}-8 \mathrm{~d}^{* *}$ & 1 & 47 & 98 \\
\hline \multirow[t]{4}{*}{$R_{1} R_{2} R_{3}$} & $1 \mathrm{a}$ & 437 & 0 & 0 \\
\hline & $2 \mathrm{~b}$ & 210 & 6 & 3 \\
\hline & $2 \mathrm{c}$ & 956 & 22 & 2 \\
\hline & $4 \mathrm{c}$ & 188 & 0 & 0 \\
\hline
\end{tabular}

* : Fresh $\rho^{+}$single colony isolate from plate

** : Segregants from a cross, $(\mathrm{d}-2) \times$ respiring haploid

Another point of interest in Table 3 is the production of $\rho^{-}$colonies in relatively experiment predominantly only among genic RD yeasts.

$\rho^{-}$mutants do not revert to respiring yeast. Hence, if there is no difference in the growth rate or selective pressure between the $\rho^{+}$and $\rho^{-}$genic mutants, the above result may be simply ascribed to biased accumulation of $\rho^{-}$cells which may be produced in a rate of one to two per cent as in the normal yeasts, because strains in question have been subjected to serial transfers prior to the examination. Such possibility, however, may be ruled out, judging from the fact that fresh $\rho^{+}$ colony just isolated from a plate, when re-spread on nutrient agar, proved to contain many $\rho^{-}$cells in nearly the same frequency as that observed in the culture of the transferred parent strain. cytoplasmic factor occurred in this 
high and low frequencies from strains of identical genotype, e.g. $4 \mathrm{~d}$ and $1 \mathrm{~d}$. That the nucleic acids metabolism and proteins metabolism may participate in promoting or repressing the change of $\rho^{+}$to $\rho^{-}$may be strongly suggested by the induction of RD yeasts by U. V. radiation at $2565 \AA$ (Raut 1953) or by the treatment with $\mathrm{Mn}^{++}$(Sarachek 1959) and pyronine B (Yanagishima 1959); stabilization of a respirationally unstable yeast by caffeine (Nagai 1962); counteraction of adenine and guanine with acriflavine as RD yeast-inducer (Nagai et al. 1961); and the inhibiting effect of cysteine on allylglycine effective to the production of RD yeasts (Sarachek and Fowler 1959). When the cytoplasmic factor was brought to an unstable state in genic RD mutants, subtle variation in the metabolism seems likely to cause a difference in the rate of change from $\rho^{+}$to $\rho^{-}$.

Diminution in the proportion of $\rho^{-}$cells in cell population during vegetative growth to the extent of approximately one per cent or less was true of respiring diploids having various genotypes, extending from the heterozygous condition for the triple pairs $\left(R_{1} r_{1}\right.$. $\left.R_{2} r_{2} \cdot R_{3} r_{3}\right)$ to the homozygous condition due to dominants $\left(R_{1} R_{1} \cdot R_{2} R_{2} \cdot R_{3} R_{3}\right)$. Therefore, the loss or instability of the cytoplasmic factor in genic mutants was concluded as a recessive character.

Thus, it may be generally accepted that the mutation of respiratory genes facilitates the induction of loss or instability of the cytoplasmic factor. Increase in mutability of the cytoplasmic factor by the lack of aerobic mechanism is suggested by the facts that (1) the production of RD yeasts from respiring yeasts is enhanced by anaerobic cultivation (Lindegren and Hino 1957), (2) the rate of induction of RD yeasts by allylglycine, $\mathrm{U}$. V. radiation, $\mathrm{Mn}^{++}$or acridine dyes is higher in anaerobically grown cells than in aerobically adapted cells (Sarachek and Fowler 1959), and (3) the cytoplasmic mutation by U.V. light is photoreactivated in the case of respiring yeasts but not in the case of genic RD yeasts (Pittman et al. 1960). However, the association of $\rho^{-}$with recessive gene $r_{2}$, but not always with recessive genes $r_{1}$ and $r_{3}$ (non-monistic relation of recessive genes to the cytoplasmic mutation) indicates that the mutability of the cytoplasmic factor in genic RD yeast cannot merely be ascribable only to the lack of aerobic mechanism per se. This view is supported by the observation that the complete loss of the cytoplasmic factor took place in three out of $17 \rho^{+}$genic RD segregants, but in none of 19 segregants of $R_{1} R_{2} R_{3} \rho^{+}$(Both of the groups of segregants came from 10 asci of a cross of (d-2) $\times$ respiring haploid), after a fairly long (about one year) preservation at $3^{\circ} \mathrm{C}$ without subculture on nutrient agar slants which were kept anaerobic by covering with liquid paraffin to the neck of slant tubes.

Since the cytoplasmic factor is regarded as auto-reproducing, the products of dominant respiratory genes (that of gene $R_{2}$ is essential) may contribute to the regulation of cellular conditions to be suitable for keeping stable the auto-reproduction of a newly formed factor for many cell generations and for keeping the rate of the auto-reproduction synchronous with the cell multiplication. 


\section{SUMMARY}

Frequent production of $\mathrm{RD}$ segregants was observed in the asci of tetraploid bakers' yeasts. With the intention of elucidating this phenomenon, genetic analysis was attempted. It was demonstrated that the respiration of the given bakers' yeasts is controlled by at least three complementary genes (tentatively denoted as $R_{1} / r_{1}, R_{2} / r_{2}$ and $R_{3} / r_{3}$ ). Heterozygous conditions containing many recessive alleles and segregation of them may account for the peculiar phenomenon in question. RD yeasts due to mutation of each of these respiratory genes were found to be characterized by a loss or instability of the cytoplasmic factor necessary for respiration. In other words, it was confirmed that the presence of the cytoplasmic factor in the yeasts concerned is stable by the existence of dominant genes, $R_{1}, R_{2}$ (indispensable) and $R_{3}$.

\section{ACKNOWLEDGMENTS}

The author wishes to express his sincerest thanks to Professor Akira Yuasa, Professor Yonosuke Ikeda and Assistant Professor Hiuga Saito of University of Tokyo. Thanks are also due to Dr. Takashi Sugimura of Research Institute of National Cancer Research for the determination of absorption spectrum and valuable advice. The author also thanks for the encouragement and support of the ex-Vice-President Tokuji Hashimoto and the staff of the Dai-Nippon Sugar Manufacturing Co., Ltd. and the technical assistance of coworkers.

\section{LITERATURE CITED}

Ephrussi, B., 1953 Nucleo-cytoplasmic relations in microorganisms: Their bearing on cell heredity and differentiation. Oxford Univ. Press, London and New York.

Ephrussi, B., and H. Hottinguer, 1951 Cytoplasmic constituents of heredity on an unstable cell state in yeast. Cold Spring Harbor Symp. Quant. Biol. 16: 75-85.

Ephrussi, B., H. Hottinguer, and H. Roman, 1955 Suppressiveness: A new factor in the genetic determinism of the synthesis of respiratory enzymes in yeast. Proc. Natl. Acad. Sci. U. S. 41: 10651071.

Gunge, N., 1966 Breeding of bakers' yeast. Determination of the ploidy and an attempt to improve practical properties. Japan. J. Genetics 41: 203-214.

Lindegren, C.C., 1955 Non-Mendelian segregation in a single tetrad of Saccharomyces ascribed to gene conversion. Science 121: 605-607.

Lindegren, C.C., and S. Hino, 1957 The effect of anaerobiosis on the origin of respiratory deficient yeast. Exptl. Cell Res. 12: 163-168.

Lindegren, C.C., and G. Lindegren, 1956 Effect of the local chromosomal environment upon the genotype. Nature 178: 796-797.

Nagai, S., 1959 Induction of the respiration-deficient mutation in yeast by various synthetic dyes. Science 130: 1188-1189.

Nagai, S., 1962 Stabilizing effect of caffeine on a respirationally unstable strain of yeast. Exptl. Cell Res. 26: 253-259. 
Nagai, S., and H. Nagai, 1958 Demonstration of the induction of respiration-deficient yeast by manganese using a selective culture medium. Naturwiss. 45: 441-442.

Nagai, S., N. Yanagishima, and H. Nagai, 1961 Advances in the study of respiration-deficient (RD) mutation in yeast and other microorganisms. Bacteriol. Rev. 25: 404-426.

Ogur, M., C. C. Lindegren, and G. Lindegren, 1954 Simple screening test for genetic studies of respiration deficiency in yeast. J. Bacteriol. 68: 391-392.

Ogur, M., and R. St. John, 1956 A differential and diagnostic plating method for population studies of respiration deficiency in yeast. ibid. 72: 500-504.

Pittman, D., J. M. Webb, A. Roshanmanesh, and L.E. Coker, 1960 Evidence for the genetic control of photoreactivation. Genetics 45: 1023-1037.

Raut, C., 1953 A cytochrome deficient mutant of Saccharomyces cerevisiae. Exptl. Cell Res. 4: 295305.

Sarachek A., 1959 The niduction by $\mathrm{Mn}^{++}$of heritable respiratory deficiency in non-dividing populations of Saccharomyces. Biochim. Biophys. Acta 33: 227-230.

Sarachek, A., and G. L. Fowler, 1959 The induction by allylglycine of heritable respiratory deficiency in Saccharomyces and its reversal by sulphur amino acids. Can. J. Microbiol. 5: 583-588.

Sherman, F., 1963 Respiration-deficient mutants of yeast. I. Genetics. Genetics 48: 375-385.

Winge, Ö., 1957 Remarks on irregular segregations in Saccharomyces. Genetics 28: 489-496.

Winge, Ö., and C. Roberts, 1950 Non-Mendelian segregation from heterozygotic yeast asci. Nature 165: $157-158$.

Winge, Ö., and C. Roberts, 1954a Causes of deviations from $2: 2$ segregations in the tetrads of monohybrid yeasts. Compt. Rend. Trav. Lab. Carlsberg, Sér. Physiol. 25: 285-329.

Winge, Ö., and C. Roberts, 1954b On tetrad analyses apparently inconsistent with Mendelian law. Heredity 8: 295-304.

Yanagishima. N., 1959 Induction of a respiration-deficient variation in yeast by pyronine B. Naturwiss. 46: 151-152.

Yčas, M., and T. Starr, 1953 The effect of glycine and protoporphyrin on a cytochrome deficient yeast. J. Bacteriol. 65: 83-88. 